\title{
PERFORMANCE ASSESSMENT OF AN INDUSTRIAL SYSTEM (AFRICAN TEXTILE MANUFACTURERS, LTD.) THROUGH COPULA LINGUISTIC APPROACH
}

This paper deals with the performance assessment of a textile industry, namely African Textile Manufactures (ATM) Ltd., in Chalawa, Kano industrial estate, Federal Republic of Nigeria. The entire system ATM has different sections of machines for processing various assignments. The machines in the sections have diverse capabilities. We assumed the following subsystems: spinning, weaving, pretreatment, printing, and steaming. The failure rates of subsystems are constant, and they follow exponential distribution, while the repairs follow two types of distributions, namely general distribution and Gumbel-Hougaard family copula distribution. The system analyzed by employing supplementary variable and the Laplace transform. Various measures of reliability have been computed for differential values of failures and two types of repair. The conclusions and some recommendations are addressed to improving the management of ATM.

Keywords: availability, reliability, MTTF, cost analysis, supplementary variables

\section{Introduction}

From the commercial to life-critical applications, the proliferation of computing systems in everyday life has substantially increased our dependence on them. Failures in the air traffic control system, nuclear reactors, or hospital patient monitoring system can bring catastrophic consequences. To enhance the dependability of computing systems, practical evaluation of their reliability is desired. With the growing complexity of

\footnotetext{
${ }^{1}$ Department of Mathematics, Kano University of Science and Technology, Wudil, Kano State, Nigeria, e-mail address: abkidris@yahoo.com

${ }^{2}$ Department of Mathematics, Yusuf Maitama Sule University, Kano State, Nigeria, e-mail address: singh_vijayvir@yahoo.com
} 
industrial systems, it is hard for management decision-makers to get accurate assessment within a predefined precision. Time to time maintenance for any reparable system is needed for successful operations. The assessment of the industrial systems through reliability measures can play a crucial role in the operational stage to predict the future behavioral aspects of the system. In the study of repairable systems, availability indices are always paramount as they directly associate with the profit incurred by the system. Therefore, researchers have adopted different types of policies to repair the system in minimal time. Availability analyses with different types of failure and single repair mode are widely exposed in the literature. A single repair facility that is employed to restore a failed system cannot be economical and will have a negative impact on production as well. When a partial failure is observed, the complex repairable systems can be restored by employing single repair, but when it fails/damaged completely, the system must be repaired using multi-dimension repair.

Many researchers presented appreciated work by considering various types of complex mathematical models and evaluated the performance of the design system using different techniques. To refer to a few, Gheisari and Goli [1] study a multi-stage $(r, s)$ -out-of- $n$ systems with dependent components. Lado et al. [2,3] study the performance and cost analysis of the repairable complex system with two subsystems in the series configuration employing general distribution and Gumbel Hougaard family copula distribution. Rawal et al. [4] studied the reliability measures of a local area network via a copula linguistics approach. Kumar and Singh [5] analyzed complex repairable systems with deliberate failure, emphasizing reboot delay using supplementary variable techniques. Rawal et al. [6] study the reliability measures of an internet data center with different types of failure and two types of repair via a copula approach. Garg [7] analyzes the reliability of an industrial system using Fuzzy Kolmogorov's differential equation a case study of a thermal power plant for evaluating the fuzzy reliability of the system. Garg et al. [8] examined a system of a pulping unit in a paper plant through preventive maintenance. Yusuf et al. [9] focus on the availability of a consecutive threestage deteriorating standby system under maintenance and replacement strategy using first-order linear differential equations. Kaur et al. [10] study a complex system of a textile industry under a preventively maintained policy using Kolmogorov differential equations. Gahlot et al. [11] investigate a system with two subsystems in series configuration under $k$-out-of- $n$ : $G$ working policy under different types of failures and two types of repair, using copula linguistic approach. Niwas and Garg [12] analyze the availability, reliability, and profit of an industrial system based on cost-free warranty policy. Qiu and Qui [13] study reliability measures, including availability analysis for repairable systems with a repair time threshold. Qiu et al. [14] examine availability and maintenance for a system subject to multiple failures, and their special case study focused on the remote power feeding system (RPFS). Qiu et al. [15] also consider the reliability parameters of maintenance models for a system subject to dependent hard and soft failure, 
using reliability density function with special emphasis put on the case study of electricity distribution. Ram et al. [17] deliberate on the stochastic analysis of a standby system with waiting for a repair strategy. Taj et al. [18] show the performance of a cable plant subsystem with priority repair over preventive maintenance, using a semi Markov process and regenerative point technique.

Most of the researchers study the reliability characteristics of a repairable complex system under the assumption that the failure can be repaired by one type of repair, but in real life situation where we observe that more than one repair is required at a time for quick maintenance of a complete failure system. In such type of situation we observe that the system needs to be repaired using joint probability distribution copula [16]. Employee strike is usually observed in the industries/ organizations: hence failure in the industrial systems cannot be ignored and due to which the system bears completely shut down mode. Realising this important fact, Singh and Rawal [19] analyse the performance of an $n$-unit's plant under different types of failure and two types of repair using copula. The preemptive resume repair policy is very effective and economic policy of reconstruction in which the repair resumes where it was left. Singh et al. [20] evaluate the performance of a system which has three units at super-priority, priority, and ordinary unit under the preemptive resume repair policy using supplementary variable techniques. Singh and Rawal [21] examine availability, MTTF, and cost analysis of the complex system under preemptive resume repair policy using copula distribution.

The authors present work with different suggestions for the improvement of performance of the repairable system, but still, some more work is required for better performance of the system. With a view on the above discussion, we have studied a reliability model, namely an African textile manufacturer (ATM), which is situated at the Challawa industrial estate at Kano state, Federal Republic of Nigeria. In the ATM, we have different types of units with various types of failures.

Copula. A $d$-dimensional copula is a distribution function on $[0,1]^{d}$ with standard uniform marginal distributions. Let $C(u)=C\left(u_{1}, \ldots, u_{d}\right)$ be the distribution functions which are copulas. Hence $C$ is a mapping of the form $C:[0,1]^{d} \rightarrow[0,1]$, i.e., a mapping of the unit hypercube into the unit interval. The following three properties must hold:

1. $C\left(u_{1}, \ldots, u_{d}\right)$ is increasing in each component $u_{i}$.

2. $C\left(1, \ldots, 1, u_{i}, 1, \ldots, 1\right)=u_{i}$ for all $i \in\{1, \ldots, d\}, u_{i} \in[0,1]$.

3. For all $\left(a_{1}, \ldots, a_{d}\right),\left(b_{1}, \ldots, b_{d}\right) \in[0,1]$ with $a_{i} \leq b_{i}$, we have: where $u_{j 1}=a_{j}$ and $u_{j 2}=b_{j}$ for all $j \in\{1, \ldots, d\}$.

The copulas are multivariate distribution functions whose one-dimensional margins are uniform on the interval $[0,1]$. The copula (joint probability distribution) approach is very natural when a complex system repaired in a couple of ways. For $\theta=1$, the Gumbel-Hougaard copula $\left(C_{\theta}\left(u_{1}, u_{2}\right)=\exp \left(-\left(\left(-\log u_{1}\right)^{\theta}+\left(-\log u_{2}\right)^{\theta}\right)^{1 / \theta}, 1 \leq \theta \leq \infty\right)\right.$, the Gumbel-Hougaard copula models become independent, and for $\theta \rightarrow \infty$ it converges 
comonotonically. Although different copulas are employed by various researchers, for conventional simplicity purpose the Gumbel-Hougaard family copula is employed for assessing the analytical cases of the paper.

\section{A brief description of the ATM system}

The production system of African textile manufacturers (ATM) is divided into five subsystems for which the present analysis is shown in Fig. 1. We discuss in brief the functioning of these subsystems.

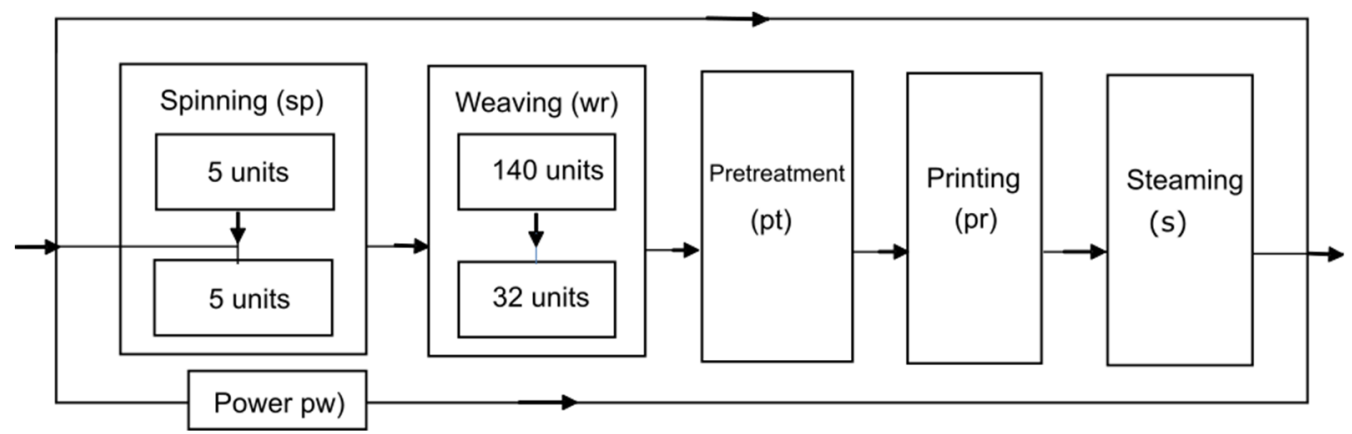

Fig. 1. ATM system configuration

\subsection{Production process}

Spinning unit (sp) - spinning is the first step of textile product processing, namely the process of making yarns from the textile fiber. This subsystem has ten engines/units, subject to the condition that if five units are working, the subsystem fulfills the requirement of the next process. When more than five unit fails, it brings the complete fail state as defined by the policy.

Weaving unit (wr) - weaving is a method of textile production in which two distinct sets of yarns or threads are interlaced at right angles to form a fabric or cloth. Out of 172 units, 140 are required to work to fulfill the next process.

Pretreatment unit (pt) - pretreatment is a heat of processing of textiles. In the pretreatment, all the impurities are removed, and fibers included in it. The failure of this unit completely fails the subsystem.

Printing unit (pr) - printing is a process of decorating textile fabrics by applying pigments, dyes, or other related materials in the form of a pattern. Failure of this unit leads to complete failure. 
Steaming (st) - steaming is one of the most essential processes in textile processing, such as wellbeing widely used in pretreatment, dyeing, finishing and printing processes, also, a failure of this stage or unit leads to complete failure.

\subsection{State description}

$\mathrm{S}_{0}$ - a perfect state where all subsystems are in good working condition.

$\mathrm{S}_{1}-$ the subsystem sp that is spinning is at degraded condition, and repair is being employed to the failed units.

$\mathrm{S}_{2}$ - the system has completely failed due to the failure of the subsystem sp.

$\mathrm{S}_{3}$ - the subsystem wr that is weaving is at degraded condition, and repair is employed.

$\mathrm{S}_{4}$ - the system is completely failed due to power failure.

$\mathrm{S}_{5}$ - the system completely failed due to unmeasurable conditions such as an earthquake, tsunami, flooding, etc. (cf. Fig. 2).

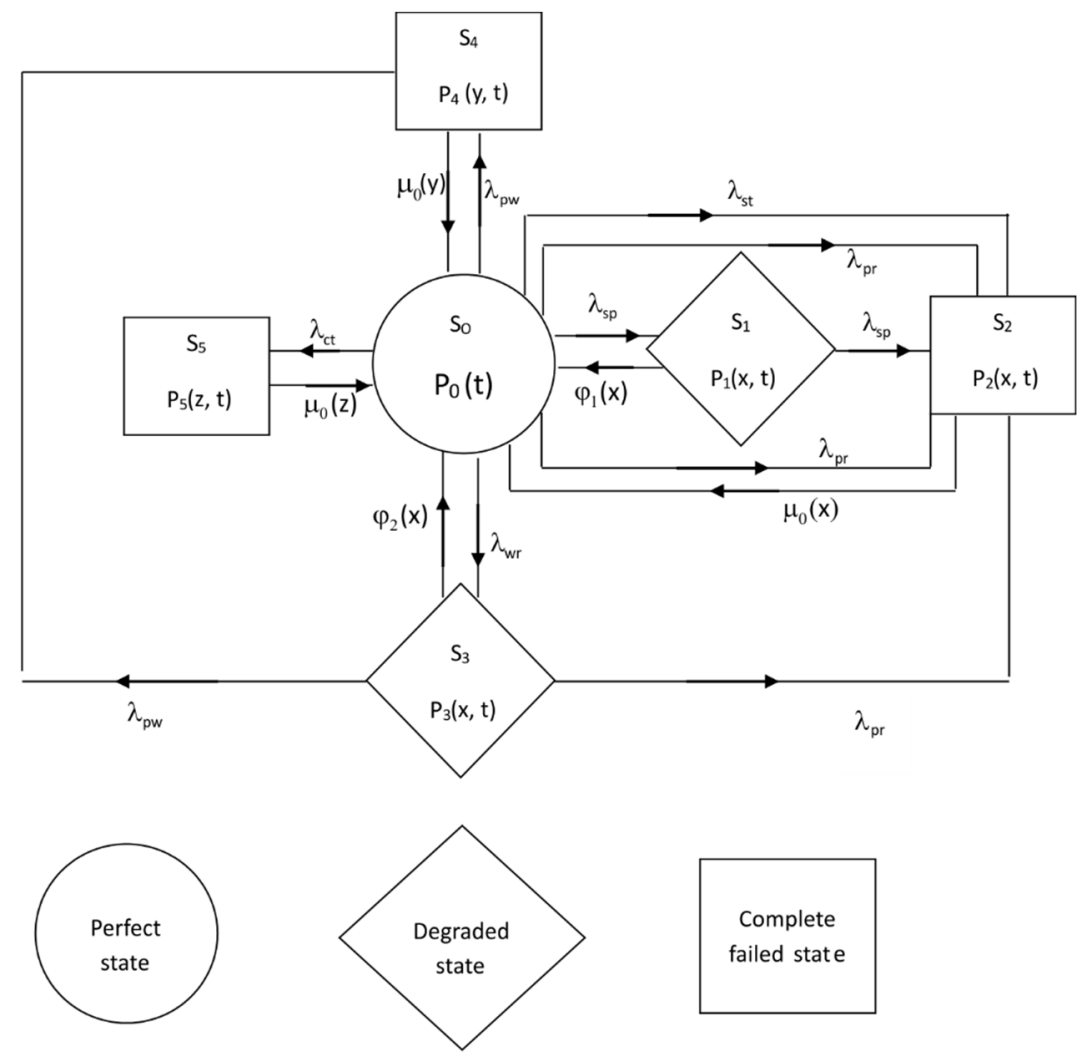

Fig. 2. State transition diagram of ATM 


\subsection{Empirical observations}

The following observations are considered throughout the discussion of the model.

- The spinning subsystem contains 10 units and if 5 units are working, the system is in the degraded state; if more than five units are working, the system is in a perfect state and fewer than five units are working means complete failure of the system.

- The weaving subsystem contains 172 units. If 140 units are working, the subsystem is in a degraded state; more than 140 units are working, means that the subsystem is in the perfect state, and fewer than 140 units is a completely failed state.

- In this stage, in the pretreatment subsystem, failure of the unit is a complete failure of the subsystem.

- Printing subsystem unit; if the unit fails, then the subsystem is seen as completely failed.

- The subsystem failed completely due to the policy defined for them.

- The system can be repaired when it is in a degraded state or a completely failed state.

- The repaired system works like a new system, and there will be no damage done due to repair.

- Power/complete failure the system needs immediate action.

- There is a constant assumption of catastrophic failure, which brings the system to a state of complete failure.

- The system block diagram is working both in series and parallel.

- As soon as the failed unit gets repaired, it starts to perform its task immediately.

\subsection{Notation used for mathematical expressions}

$\lambda_{s p}, \lambda_{w r}, \lambda_{p t}, \lambda_{p r}, \lambda_{s t}$ - failure rates of the spinning, weaving, pretreatment, printing, steaming of the subsystems, respectively.

$\lambda_{p w}, \lambda_{c t}$ - failure rates due to power, catastrophic, respectively.

$\varphi_{1}(x), \varphi_{2}(x), \mu_{0}(x), \mu_{0}(y), \mu_{0}(z)$ - repair rates for spinning, weaving, pretreatment, printing, steaming power, catastrophic, respectively.

$P_{i}(t)$ - the probability that the system is in $\mathrm{S}_{i}$ state at $t$ for $i=0, \ldots, 5$.

$s$ - Laplace transform variable.

$\bar{P}_{i}(s)$ - Laplace transformation of $P(t)$.

$P_{i}(x, t)$ - the probability that the system is in the state $\mathrm{S}_{0}$ for $i=1-5$, the system running under repair and elapsed repair time is $(x, t)$.

$E_{p}(t)$ - expected profit during the interval $[0, t)$.

$K_{1}, K_{2}$ - revenue and service cost per unit time, respectively. 
$\mu_{0}(x)=C_{\theta}\left(u_{1}(x), u_{2}(x)\right)$ - the expression of joint probability (failed state $\mathrm{S}_{i}$ to good state $\mathrm{S}_{0}$ ) according to Gumbel-Hougaard family copula is

$$
C_{\theta}\left(u_{1}(x), u_{2}(x)\right)=\exp \left[x^{\theta}+\{\log \varphi(x)\}^{\theta}\right]^{1 / \theta}
$$

where $u_{1}=\varphi(x)$, and $u_{2}=\mathrm{e}^{x}$, where $\theta$ is a parameter, $1 \leq \theta \leq \infty$.

\section{Formulation and solution of the mathematical model}

\subsection{Formulation of the model}

By the probability of considerations and continuity of arguments, the following set of difference-differential equations is associated with the present mathematical model:

$$
\begin{gathered}
{\left[\frac{\partial}{\partial t}+\lambda_{1}+\lambda_{2}+\lambda_{3}+\lambda_{4}+\lambda_{5}+\lambda_{6}+\lambda_{7}\right] p_{0}(t)=\int_{0}^{\infty} \varphi_{1}(x) p_{1}(x, t) d x} \\
+\int_{0}^{\infty} \varphi_{2} p_{3}(x, t) d x+\int_{0}^{\infty} \mu_{0}(x) p_{2}(x, t) d x+\int_{0}^{\infty} \mu_{0}(y) p_{4}(\mathrm{y}, t) d y+\int_{0}^{\infty} \mu_{0}(z) p_{5}(z, t) d z \\
{\left[\frac{\partial}{\partial t}+\frac{\partial}{d x}+\lambda_{1}+\lambda_{6}+\varphi_{1}(x)\right] p_{1}(x, t)=0} \\
{\left[\frac{\partial}{\partial t}+\frac{\partial}{d x}+\mu_{0}(x)\right] p_{2}(x, t)=0} \\
{\left[\frac{\partial}{\partial t}+\frac{\partial}{\partial y}+\lambda_{2}+\lambda_{6}+\varphi_{2}(x)\right] p_{3}(x, t)=0} \\
{\left[\frac{\partial}{\partial t}+\frac{\partial}{\partial y}+\mu_{0}(y)\right] p_{4}(y, t)=0} \\
{\left[\frac{\partial}{\partial t}+\frac{\partial}{\partial z}+\mu_{0}(z)\right] p_{5}(z, t)=0}
\end{gathered}
$$


Boundary conditions

$$
\begin{gathered}
p_{1}(0, t)=\lambda_{1} p_{0}(t) \\
p_{2}(0, t)=\lambda_{1} p_{1}(0, t)+\lambda_{2} p_{3}(0, t)+\lambda_{3} p_{0}(0, t)+\lambda_{4} p_{0}(0, t)+\lambda_{5} p_{0}(0, t) \\
p_{3}(0, t)=\lambda_{2} p_{0}(t) \\
p_{2}(0, t)=\left(\lambda_{1} \lambda_{1}+\lambda_{2} \lambda_{2}+\lambda_{3}+\lambda_{4}+\lambda_{5}\right) p_{0}(t) \\
p_{4}(0, t)=\lambda_{6} p_{0}(t)+\lambda_{6} p_{1}(0, t)+\lambda_{6} p_{3}(0, t) \\
P_{4}(0, t)=\left(\lambda_{6}+\lambda_{1} \lambda_{6}+\lambda_{2} \lambda_{6}\right) P_{0}(t) \\
p_{4}(0, t)=\lambda_{7} p_{0}(t)
\end{gathered}
$$

$p_{0}(0)=1$ and other state transition probabilities at $t=0$ are zero

\subsection{Solution of the model}

Taking Laplace transformation of equations (1)-(11) and using (12), one can obtain.

$$
\begin{gathered}
{\left[s+\lambda_{1}+\lambda_{2}+\lambda_{3}+\lambda_{4}+\lambda_{5}+\lambda_{6}+\lambda_{7}\right] \bar{P}_{0}(s)=1+\int_{0}^{\infty} \varphi_{1}(x) \bar{P}_{1}(x, s) d x} \\
+\int_{0}^{\infty} \varphi_{2}(x) \bar{P}_{3}(x, s) d x+\int_{0}^{\infty} \mu_{0}(x) \bar{P}_{2}(x, s) d x \\
+\int_{0}^{\infty} \mu_{0}(y) \bar{P}_{4}(y, s) d y+\int_{0}^{\infty} \mu_{0}(z) \bar{P}_{5}(y, s) d z \\
{\left[s+\frac{\partial}{\partial x}+\lambda_{2}+\lambda_{6}+\varphi_{1}(x)\right] \bar{P}_{1}(x, s)=0} \\
{\left[s+\frac{\partial}{\partial x}+\mu_{0}(x)\right] \bar{P}_{2}(x, s)=0} \\
{\left[s+\frac{\partial}{\partial x}+\lambda_{2}+\lambda_{6}+\varphi_{2}(x)\right] \bar{P}_{3}(x, s)=0}
\end{gathered}
$$




$$
\begin{aligned}
& {\left[s+\frac{\partial}{\partial y}+\mu_{0}(y)\right] \bar{P}_{4}(y, s)=0} \\
& {\left[s+\frac{\partial}{\partial z}+\mu_{0}(z)\right] \bar{P}_{5}(z, s)=0}
\end{aligned}
$$

Laplace transform of boundary conditions

$$
\begin{gathered}
\bar{P}_{1}(0, s)=\lambda_{1} \bar{P}_{0}(s) \\
\bar{P}_{2}(0, s)=\left(\lambda_{1} \lambda_{1}+\lambda_{2} \lambda_{2}+\lambda_{3}+\lambda_{4}+\lambda_{5}\right) \bar{P}_{0}(s) \\
\bar{P}_{3}(0, s)=\lambda_{2} \bar{P}_{0}(s) \\
\bar{P}_{4}(0, s)=\left(\lambda_{6}+\lambda_{1} \lambda_{6}+\lambda_{2} \lambda_{6}\right) \bar{P}_{0}(s) \\
\bar{P}_{5}(0, s)=\lambda_{7} \bar{P}_{0}(s)
\end{gathered}
$$

Solving (14)-(18), with the help of boundary conditions (19) to (23) one may get,

$$
\begin{gathered}
\bar{P}_{0}(s)=\frac{1}{D(s)} \\
\bar{P}_{1}(s)=\frac{\lambda_{1}}{D(s)}\left\{\frac{1-\bar{S}_{\varphi_{1}}\left(s+\lambda_{1}+\lambda_{6}\right)}{s+\lambda_{1}+\lambda_{6}}\right\} \\
\bar{P}_{2}(s)=\left(\frac{\left(\lambda_{1} \lambda_{1}+\lambda_{2} \lambda_{2}+\lambda_{3}+\lambda_{4}+\lambda_{5}\right)}{D(s)}\right)\left(\frac{1-\bar{S}_{\mu_{0}}(x)(s)}{s}\right) \\
\bar{P}_{3}(s)=\frac{\lambda_{2}}{D(s)}\left\{\frac{1-\bar{S}_{\varphi_{2}}\left(s+\lambda_{1}+\lambda_{6}\right)}{s+\lambda_{1}+\lambda_{6}}\right\} \\
\bar{P}_{4}(s)=\left(\frac{\left(\lambda_{6}+\lambda_{1} \lambda_{6}+\lambda_{2} \lambda_{6}\right)}{D(s)}\right)\left\{\frac{1-\bar{S}_{\mu_{0}}(s)}{s}\right\}
\end{gathered}
$$




$$
\bar{P}_{5}(s)=\frac{\lambda_{7}}{D(s)}\left\{\frac{1-\bar{S}_{\mu_{0}}(s)}{s}\right\}
$$

where

$$
\begin{aligned}
D(s)= & \left(s+\lambda_{1}+\lambda_{2}+\lambda_{3}+\lambda_{4}+\lambda_{5}+\lambda_{6}+\lambda_{7}\right) \\
& -\left(\frac{\lambda_{1} \bar{S}_{\varphi_{1}}(s)}{s+\lambda_{1}+\lambda_{6}+\varphi_{1}}+\frac{\lambda_{2} \bar{S}_{\varphi_{2}}(s)}{s+\lambda_{2}+\lambda_{6}+\varphi_{2}}+\frac{\left(\lambda_{1} \lambda_{2}+\lambda_{2} \lambda_{3}+\lambda_{3}+\lambda_{4}+\lambda_{5}\right) S_{\mu_{0}}(s)}{s+\mu_{0}}\right. \\
& \left.+\frac{\left(\lambda_{6}+\lambda_{1} \lambda_{6}+\lambda_{2} \lambda_{6}\right) S_{\mu_{0}}(s)}{s+\mu_{0}}+\frac{\lambda_{7} \bar{S}_{\mu_{0}}(s)}{s+\mu_{0}}\right)
\end{aligned}
$$

The sum of Laplace transformations of the state transition probabilities when the system is in operational and non-operational mode is as follows:

$$
\begin{gathered}
\bar{P}_{\text {up }}(s)=\bar{P}_{0}(s)+\bar{P}_{1}(s)+\bar{P}_{2}(s)+\bar{P}_{3}(s) \\
\bar{P}_{\text {down }}(s)=1-\bar{P}_{\text {up }}(s)
\end{gathered}
$$

\section{Analytical study of the model for particular cases}

\subsection{Availability analysis}

\section{A. The system follows Gumbel-Hougaard's family copula}

Setting

$$
S_{\mu_{0}}(s)=\bar{S}_{\exp \left[x^{\theta}+\{\log \varphi(x)\}^{\theta}\right]^{1 \theta}}(s)=\frac{\exp \left[x^{\theta}+\{\log \varphi(x)\}^{\theta}\right]^{1 / \theta}}{s+\exp \left[x^{\theta}+\{\log \varphi(x)\}^{\theta}\right]^{1 / \theta}}, \quad S_{\varphi_{i}}(s)=\frac{\varphi_{i}}{s+\varphi_{i}}, i=1,2
$$

and taking the values of different parameters as $\lambda_{1}=0.012, \lambda_{2}=0.013, \lambda_{3}=0.014$, $\lambda_{4}=0.015, \lambda_{5}=0.016, \lambda_{6}=0.018, \lambda_{7}=0.020, \varphi_{1}=\varphi_{2}=1, \theta=1, x=1$ in (30), and then taking the inverse Laplace transform, one can obtain, the expression for availability as: 


$$
\begin{aligned}
P_{\text {up }}(t)= & 3.07692308 \mathrm{e}^{-0.03100000000 t}+0.02914038208 \mathrm{e}^{-2.800060728 t} \\
& -0.4522002611 \mathrm{e}^{-0.1841576900 t}-1.627558692 \mathrm{e}^{-0.03047995911 t} \\
& -0.2570737371 \mathrm{e}^{-0.1273983772 t}+\mathrm{e}^{-0.30000000000 t}
\end{aligned}
$$

For different values of time variable $t=0,1,2,3,4,5,6,7,8,9, \ldots$ units of time, one may get different values of $P_{\text {up }}(t)$ with the help of (32a) as shown in Table 1, and corresponding Fig. 3.

Table 1. Availability in dependence of time units

\begin{tabular}{|l|c|c|c|c|c|c|c|c|c|c|}
\hline Time $(t)$ & 0 & 1 & 2 & 3 & 4 & 5 & 6 & 7 & 8 & \\
\hline Availability & 1.0000 & 0.9626 & 0.9349 & 0.8943 & 0.8403 & 0.7734 & 0.6938 & 0.6015 & 0.4961 & 0.3768 \\
\hline
\end{tabular}

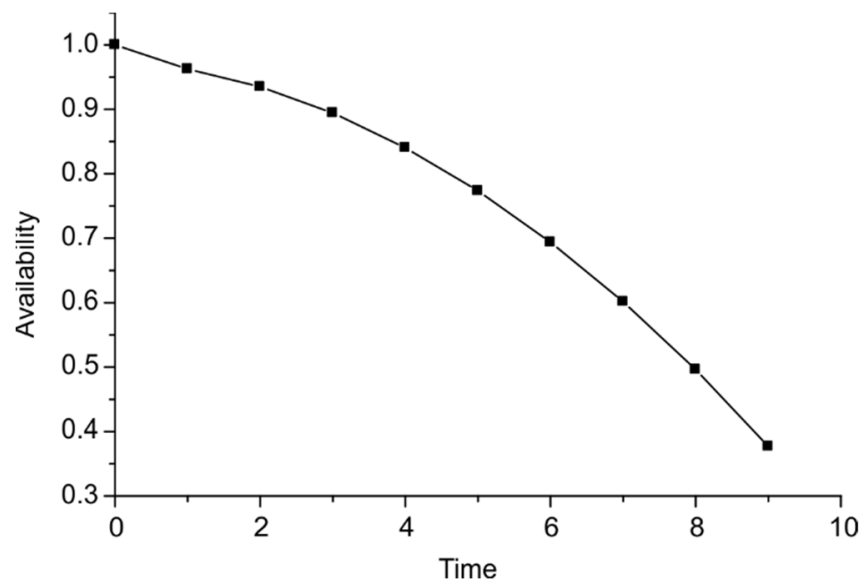

Fig. 3. Time dependence of availability (case 4.1A)

\section{B. The system follows general repair}

Setting

$$
\bar{S}_{\mu_{0}}(s)=\bar{S}_{\varphi_{i}}(s)=\frac{\varphi_{i}}{s+\varphi_{i}}, \quad i=1,2
$$

and taking the values of different parameters as $\lambda_{1}=0.012, \lambda_{2}=0.013, \lambda_{3}=0.014$, $\lambda_{4}=0.015, \lambda_{5}=0.016, \lambda_{6}=0.018, \lambda_{7}=0.020, \varphi_{1}=\varphi_{2}=1, \theta=1, x=1$, in (30), and then taking the inverse Laplace transform, one can obtain, the expression for availability as: 


$$
\begin{aligned}
P_{\text {up }}(t)= & 2.306792308 \mathrm{e}^{-0.0310000000 t}-0.02745133242 \mathrm{e}^{-0.1247551404 t} \\
& +0.07644883675 \mathrm{e}^{-0.1841576900 t}-0.4821123635 \mathrm{e}^{-0.1785923385 t} \\
& -1.627515456 \mathrm{e}^{-003047994292 t}+\mathrm{e}^{-0.30000000000 t}
\end{aligned}
$$

For different values of time variable $t=0,1,2,3,4,5,6,7,8,9, \ldots$, units of time, one may get different values of $P_{\text {up }}(t)$ with the help of (32b) as shown in Table 2 and corresponding Fig. 4. It can easily be concluded from Tables 1 and 2 that when the repair follows copula repair, the availability of the system improves and, as a result, the incurred profit also increases. Conclusively, copula repair improves the performance of the system. It should be noted that other reliability indices like system reliability and MTSF remains unaffected by copula repair (Fig. 5).

Table 2. Availability in dependence of time units

\begin{tabular}{|l|c|c|c|c|c|c|c|c|c|c|}
\hline Time $(t)$ & 0 & 1 & 2 & 3 & 4 & 5 & 6 & 7 & 8 & 9 \\
\hline Availability & 1.0000 & 0.9406 & 0.8986 & 0.8531 & 0.7976 & 0.7303 & 0.6505 & 0.5578 & 0.4519 & 0.3319 \\
\hline
\end{tabular}

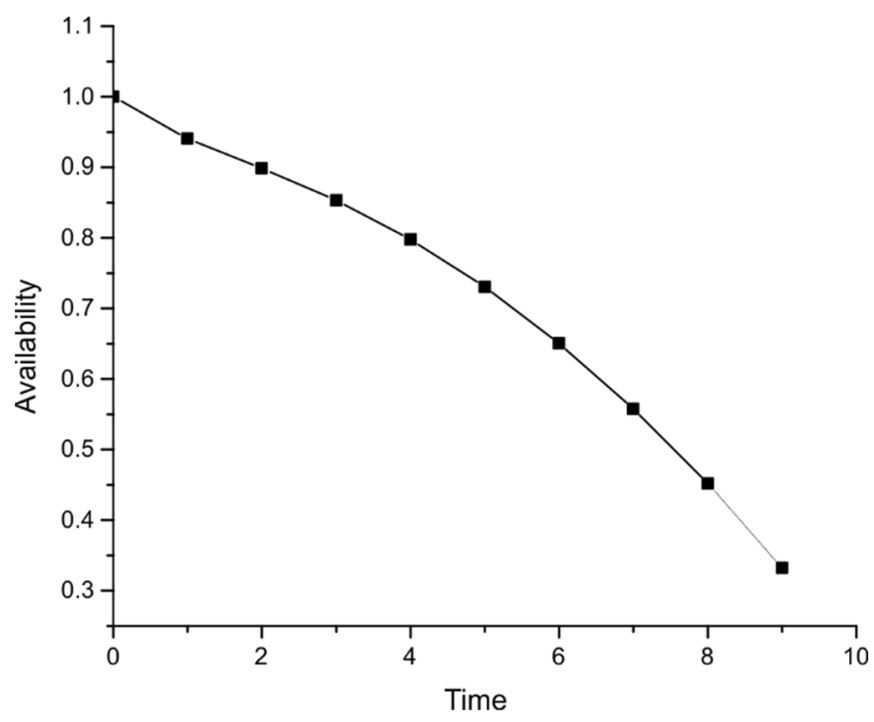

Fig. 4. Time dependence of availability (case 4.1B) 


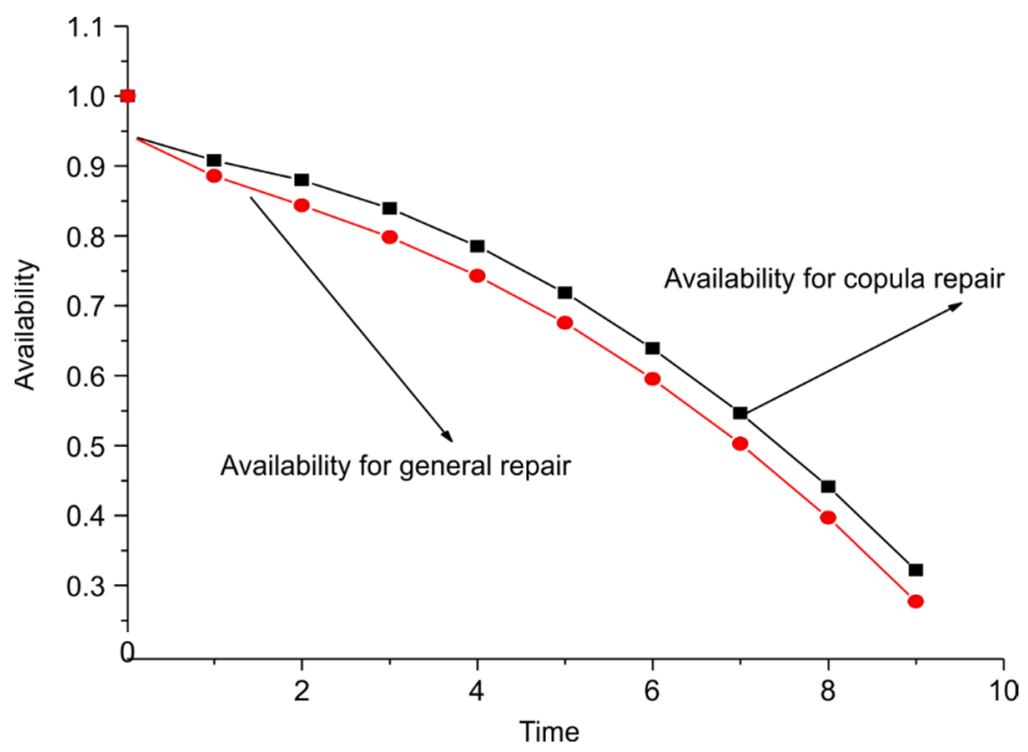

Fig. 5. Availability comparison (with copula repair and without copula repair)

\subsection{Reliability analysis}

Taking all repair rates, $\varphi_{1}(x), \varphi_{2}(x), \mu_{0}(x), \mu_{0}(y), \mu_{0}(z)$ in equation (30) to zero and for same values of failure rates as $\lambda_{1}=0.012, \lambda_{2}=0.013, \lambda_{3}=0.014$, $\lambda_{4}=0.015, \lambda_{5}=0.016, \lambda_{6}=0.018, \lambda_{7}=0.020, \varphi_{1}(x)=\varphi_{2}(x)=1, \theta=1, x=1$ in (30), and then taking inverse Laplace transform, one may arrive at the expression for reliability for the system:

$$
\begin{aligned}
R(t)= & \left\{0.1688311688 \mathrm{e}^{-0.3100000000 t}+0.6773226773 \mathrm{e}^{-0.1080000000 t}\right. \\
& \left.+0.1538461538 \mathrm{e}^{-0.0300000000 t}\right\}
\end{aligned}
$$

For different values of time $t=0,1,2,3,4,5,6,7,8,9, \ldots$, units of time, one may get different values of reliability as shown in Table 3 and graphical representation in Fig. 6.

Table 3. Computation of reliability with respect to time

\begin{tabular}{|l|c|c|c|c|c|c|c|c|c|c|}
\hline Time $(t)$ & 0 & 1 & 2 & 3 & 4 & 5 & 6 & 7 & 8 & 9 \\
\hline Availability & 1.0000 & 0.9209 & 0.8493 & 0.7843 & 0.7253 & 0.6717 & 0.6229 & 0.5786 & 0.5382 & 0.5014 \\
\hline
\end{tabular}




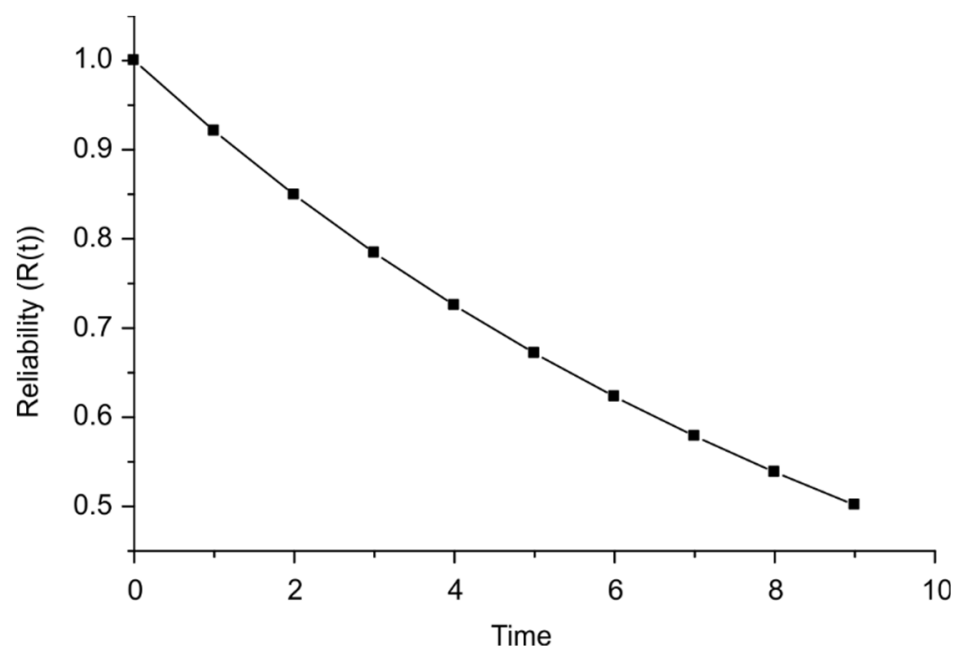

Fig. 6. Time dependence of reliability

\subsection{Mean time to failure (MTTF) analysis}

Taking all repairs to zero in equation (30), and the taking limit as $s$ tending to zero one can obtain the expression for MTTF:

$$
\mathrm{MTTF}=\lim _{s \rightarrow 0} \bar{P}_{\text {up }}(s)=\frac{1}{\lambda_{1}+\lambda_{2}+\lambda_{3}+\lambda_{4}+\lambda_{5}+\lambda_{6}+\lambda_{7}}\left\{1+\frac{1}{\lambda_{1}+\lambda_{2}}+\frac{}{\lambda_{2}+\lambda_{6}}\right\}
$$

Table 4. Computed MTTF values corresponding to various failure rates

\begin{tabular}{|c|c|c|c|c|c|c|c|}
\hline Failure rate & $\lambda_{1}$ & $\lambda_{2}$ & $\lambda_{3}$ & $\lambda_{4}$ & $\lambda_{5}$ & $\lambda_{6}$ & $\lambda_{7}$ \\
\hline 0.1 & 16.7594 & 16.7346 & 17.4937 & 17.6636 & 17.8368 & 21.1067 & 18.5648 \\
\hline 0.2 & 16.7730 & 16.7505 & 15.9592 & 16.1004 & 16.2442 & 16.0812 & 16.8458 \\
\hline 0.3 & 16.2250 & 16.2000 & 14.6722 & 14.7915 & 14.9127 & 13.2336 & 15.4182 \\
\hline 0.4 & 15.5074 & 15.4789 & 13.5772 & 13.6793 & 13.7829 & 11.3542 & 14.2137 \\
\hline 0.5 & 14.7578 & 14.7261 & 12.6344 & 12.7227 & 12.8123 & 9.99928 & 13.1837 \\
\hline 0.6 & 14.0293 & 13.9950 & 11.8139 & 11.8912 & 11.9694 & 8.9649 & 12.2929 \\
\hline 0.7 & 13.3422 & 13.3057 & 11.0936 & 11.1616 & 11.2305 & 8.1435 & 11.5149 \\
\hline 0.8 & 12.7027 & 12.6647 & 10.4560 & 10.5165 & 10.5776 & 7.4718 & 10.8294 \\
\hline 0.9 & 12.1112 & 12.0720 & 9.8877 & 9.9418 & 9.9964 & 6.9103 & 10.2210 \\
\hline
\end{tabular}

Setting $\lambda_{1}=0.012, \lambda_{2}=0.013, \lambda_{3}=0.014, \lambda_{4}=0.015, \lambda_{5}=0.016, \lambda_{6}=0.018$, $\lambda_{7}=0.020$ and varying from $\lambda_{1}$ to $\lambda_{7}$ one by one as $0.1,0.2,0.3,0.4,0.5,0.6,0.7,0.8$, 0.9 , respectively, one would obtain the variation of MTTF, concerning failure rates, as shown in Table 4 and corresponding Fig. 7. 


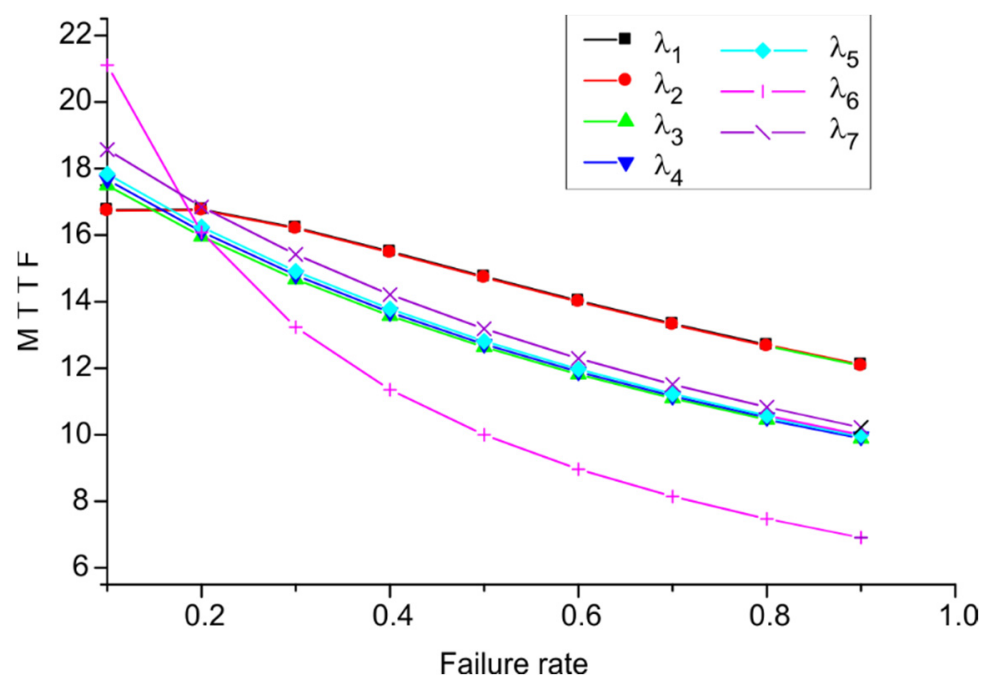

Fig. 7. MTTF in the function of failure rates

\subsection{Cost analysis}

If the service facility is always available, then expected profit during the interval $[0, t)$ of the system can be obtained from the formula

$$
E_{p}(t)=K_{1} \int_{0}^{t} P_{\text {up }}(t) d t-K_{2} t
$$

For the same set of parameters of (30), one can obtain (35). Therefore

$$
\begin{aligned}
E_{p}(t)= & -74.44168735 \mathrm{e}^{-0.03100000000 t}-0.0140705360 \mathrm{e}^{-2.800060728 t} \\
& +2.45505720 \mathrm{e}^{-0.1841576900 t} \\
& +53.39766652 \mathrm{e}^{-0.03047995911 t}-2.017872933 \mathrm{e}^{0.1273983772 t} \\
& -33.33333333 \mathrm{e}^{-0.0300000000 t}+53.95012842
\end{aligned}
$$

Setting $K_{1}=1$ and $K_{2}=0.6,0.5,0.4,0.3,0.2$ and 0.1 , respectively, and varying $t=0,1,2,3,4,5,6,7,8,9$ units of time, the results for expected profit can be obtained, as shown in Table 5 and Fig. 8. 
Table 5. Variation of expected profit $E_{P}(t)$ with respect to time in the interval $[0, t)$

\begin{tabular}{|c|c|c|c|c|c|c|}
\hline Time & $k_{2}=0.6$ & $k_{2}=0.5$ & $k_{2}=0.4$ & $k_{2}=0.3$ & $k_{2}=0.2$ & $k_{2}=0.1$ \\
\hline 0 & 0 & 0 & 0 & 0 & 0 & 0 \\
\hline 1 & 0.3770 & 0.4770 & 0.5770 & 0.6770 & 0.7770 & 0.8770 \\
\hline 2 & 0.7267 & 0.9267 & 1.1267 & 1.3267 & 1.5267 & 1.7267 \\
\hline 3 & 1.0425 & 1.3425 & 1.6425 & 1.9425 & 2.2425 & 2.5425 \\
\hline 4 & 1.3110 & 1.7110 & 2.1110 & 2.5110 & 2.9110 & 3.3110 \\
\hline 5 & 1.5189 & 2.0189 & 2.5189 & 3.0189 & 3.5189 & 4.0189 \\
\hline 6 & 1.6535 & 2.2436 & 2.8536 & 3.4536 & 4.0536 & 4.6536 \\
\hline 7 & 1.7023 & 2.4023 & 3.1023 & 3.8023 & 4.5023 & 5.2023 \\
\hline 8 & 1.6523 & 2.5523 & 3.2523 & 4.0523 & 4.8523 & 5.6523 \\
\hline 9 & 1.5990 & 2.7900 & 3.2900 & 4.1900 & 5.4900 & 5.9900 \\
\hline
\end{tabular}

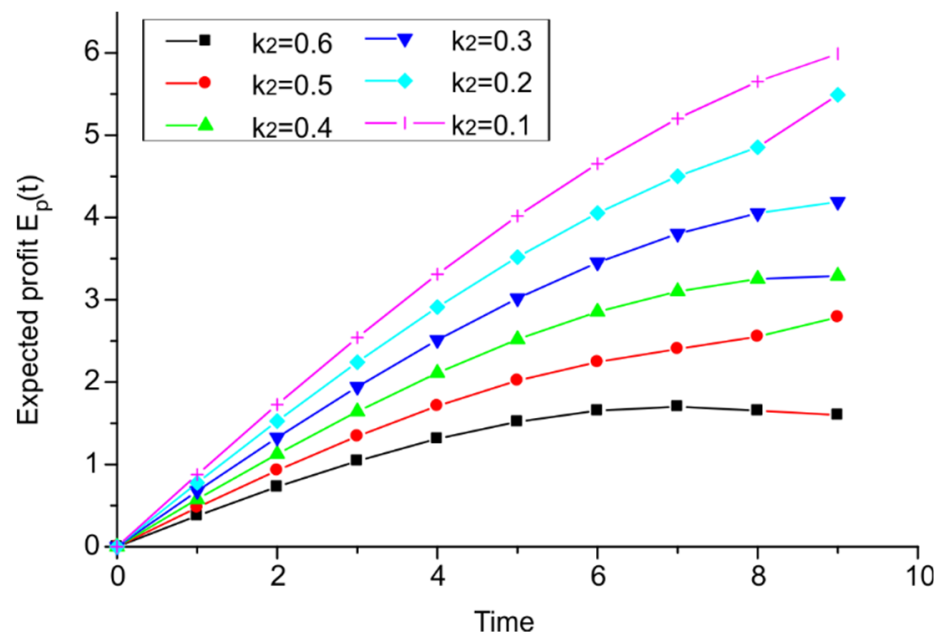

Fig. 8. Expected profit as the function of time

\section{Discussion and conclusions}

Tables 1 and 2 provide information on the availability of the complex repairable system change concerning the time when failure rates are fixed at different values. When failure rates are set at lower values $\lambda_{1}=0.012, \lambda_{2}=0.013, \lambda_{3}=0.014, \lambda_{4}=0.015$, $\lambda_{5}=0.016, \lambda_{6}=0.018, \lambda_{7}=0.020$, the availability of the system decreases as time $t$ increases and ultimately becomes steady at zero level after a sufficiently long interval of time. The values of availability are smaller when the repair follows the general distribution. Conclusively, copula repair is a more effective repair policy for better performance of repairable systems (Tables 1, 2, Figs. 3, 4). 
Based on information regarding the reliability of the system for different values of failure rates, one can safely predict the future behavior of a complex system at any time for any given set of parametric values (Table 3, Fig. 6).

The variation in MTTF corresponding to different failure rates indicates that incremental change in values of parameter decreases the MTTF of the repairable system (Table 4, Fig. 7). It is interesting to note that in this case, MTTF of the system decreases smoothly with each failure rate. The failures of $\lambda_{1}$ and $\lambda_{6}$ are more responsible for the successful operation of the system (Fig. 7). A critical examination of Table 5 and Fig. 8 reveals that the expected profit increases for the time when the service cost $K_{2}$ decreases. The computed expected profit for $K_{2}$ from 0.6 to 0.1 is maximum for $K_{2}=0.1$ and minimum at $K_{2}=0.6$. Conclusively, one can observe that as service cost decreases, profit increases upon increasing time. In general, for low service costs, the expected profit is high in comparison to the high service cost.

\section{References}

[1] GHeISARI A., Goli S., A study on the multi-state ( $r, s)$-out-of-n systems with dependent components, Int. J. Rel. Risk Saf., 2018, 1 (1), 11-16.

[2] LAdO A., Singh V.V., ISMAIL K.H., IBRAHIM Y., Performance and cost assessment of repairable complex system with two subsystems connected in series configuration, Int. J. Rel. Appl., 2018, 19 (1), 27-42.

[3] LAdO A., Singh V.V., Cost assessment of complex repairable system consisting of two subsystems in the series configuration using Gumbel-Hougaard family copula, Int. J. Qual. Rel. Manage., 2019, 36 (10), 1683-1698.

[4] Rawal D.K., RAm M., Singh V.V., Study of reliability measures of a local area network via copula linguistics approach, Int. J. Qual. Rel. Manage., Emerald, 2015, 32 (1), 97-111, 2015.

[5] Kumar D., Singh S.B., Stochastic analysis of the complex repairable system with deliberate failure emphasizing reboot delay, Comm. Stat.-Simul. Comp., 2016, 45, 1-20.

[6] RaWAl D.K., RAM M., Singh V.V., Modeling and availability analysis of internet data center with various maintenance policies, Int. J. Eng., Trans. A, Basics, 2014, 27 (4), 599-608.

[7] GARG H., An approach of analyzing the reliability of industrial system using fuzzy Kolmogorov's differential equation, Arab. J. Sci. Eng., 2015, 40, 975-987.

[8] GARG H., RANI SHARMA S.P., Preventive maintenance scheduling of the pulping unit in a paper plant, Japan J. Appl. Math., 2013, 30, 397-414.

[9] YUSUf I., Gatawa R.I., SLeIMAN K., Availability analysis of a consecutive three stages deteriorating standby system considering maintenance and replacement, J. Math. Sci. Model., 2018, 1 (1), 21-26.

[10] Kaur M., LARA A.K., BhATIA S.S., RedDY A.S., Corrective maintenance data for performance analysis of the preventively maintained textile industry, J. Rel. Stat. Studies, 2013, 6 (2), 155-163.

[11] Gahlot M., Singh V.V., Ayagi H., Goel C.K., Performance assessment of repairable system in series configuration under different types of failure and repair policies using copula linguistics, Int. J. Rel. Safe., 2018, 12 (4), 348-374.

[12] NiwAS R., GARG H., An approach for analyzing the reliability and profit of an industrial system based on the cost-free warranty policy, J. Brazil. Soc. Mech. Eng., 2018, 40, 1-9.

[13] QIU Q., CUI L., Availability analysis for general repairable systems with repair time threshold, Comm. Stat. Theory Meth., 2017, 48 (3), 1-20. 
[14] QIU Q., CUI L., GAO H., Availability and maintenance modeling for systems subject to multiple failure models, J. Comp. Ind. Eng., 2017, 108, 192-198.

[15] QIU Q., CUI L., SHEN J., Availability and maintenance modeling for systems subject to dependent hard and soft failures, Appl. Stoch. Mod. Bus. Ind., 2018, 34 (4), 513-527.

[16] Nelsen R.B., An introduction to copulas, 2nd Ed., Springer, New York 2006.

[17] Ram M., Singh S.B., Singh V.V., Stochastic analysis of a standby system with waiting repair strategy, IEEE Trans. Syst., Manage., Cyber. Syst., 2013, 43 (3), 698-707.

[18] TAj S.Z., Rizman S.M., Alkali B.M., Probabilistic modeling and analysis of a cable plant subsystem with priority to repair over preventive maintenance, Manage. J. Math., 2017, 6 (3), 13-21.

[19] SingH V.V., RAWAL D.K., Stochastic analysis of N-unit plant with different types of failure and repair policy using copula distribution, J. Rel. Stat. Stud., 2015, 8 (2), 119-133.

[20] Singh V.V., Singh S.B., RAm M., GoEl C.K., Availability of a system having three units. Superpriority, priority and ordinary under preemptive resume policy, Int. J. Rel. Appl., 2010, 11 (1), 41-53.

[21] Singh V.V., RAWAL D.K., Availability, MTTF, and cost analysis of complex system under preemptive resume repair policy using copula distribution, J. Stat. Oper. Res., 2014, 10 (3), 299-321.

Received 3 August 2019

Accepted 4 January 2020 